\title{
Alpha Cygnids - a possible July minor meteor shower
}

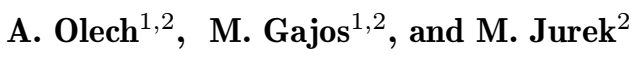 \\ 1 Warsaw University Observatory, Al. Ujazdowskie 4, 00-478 Warszawa, Poland \\ 2 Comets and Meteors Workshop, ul. Sokolicz 3/59, 01-508 Warszawa, Poland
}

Received June 23; accepted November 6, 1998

\begin{abstract}
We present a comprehensive study of a possible $\alpha$-Cygnid meteor shower. Based on visual and telescopic observations made by Polish observers we estimate basic parameters of the stream. Activity of $\alpha$-Cygnids lasts from around June 30 to July 31 with clear maximum near July 18 (solar longitude $\lambda_{\odot}=116.5^{\circ}$ ). Maximal Zenithal Hourly Rates $(Z H R \mathrm{~s})$ are equal to $3.6 \pm 1.2$. The structure of the radiant analyzed by RADIANT software is most compact for geocentric velocity of the events equal to $41 \mathrm{~km} \mathrm{~s}^{-1}$, and for the drift of the radiant (in units $\%$ day) equal to $\Delta \alpha=+0.6 \Delta \delta=+0.2$. Center of the radiant for the moment of maximum is $\alpha=302.5^{\circ} \delta=+46.3^{\circ}$. We derive population index $r$ equal to $2.55 \pm 0.14$ from magnitude distribution of 738 possible members of the stream. Comparing the velocity distributions of 754 possible $\alpha$ Cygnids and 4339 sporadic events by $\chi^{2}$ and KolmogorovSmirnov tests we conclude that both distributions are different with a probability very close to 1.0.

Telescopic observations strictly confirm the results obtained from visual observations. The smallest values of $\chi^{2}$ parameter were obtained for the geocentric velocity equal to $40 \mathrm{~km} \mathrm{~s}^{-1}$ and for the drift of the radiant (in units $\%$ day) equal to $\Delta \alpha=+0.6 \Delta \delta=+0.2$. The center of the radiant for moment of maximum is $\alpha=304.9^{\circ}$ $\delta=+46.2^{\circ}$.

In spite of making many photographic exposures we still have no photographic or video confirmation of the existence of this stream.
\end{abstract}

Key words: meteoroids, meteors — interplanetary medium

\section{Introduction}

The first data about meteors from radiant near $\alpha$ Cygni come from W.F. Denning (Denning 1919). In years

Send offprint requests to: Arkadiusz Olech, e-mail: olech@sirius.astrouw.edu.pl
1885-1918 he observed 50 meteors radiating from the close vicinity of Deneb. He did not know the activity period of the stream so he noted meteors during the whole year. It is clearly visible from his Table 2 that most of the meteors were noted during July nights. Almost all events observed this month were classified as rapid.

During the next years one can find the different parameters describing $\alpha$-Cygnids stream in the astronomical literature. Polish meteor publications were giving activity period between June 16th and July 31st. No clear maximum of activity was found (Kosinski 1990).

Photographic data are also poor. Only one possible member was captured in Dushanbe on 1961 July 12. The radiant of this event was $\alpha=304.5^{\circ}, \delta=+49.7^{\circ}$ and geocentric velocity $V_{\infty}=41.0 \mathrm{~km} \mathrm{~s}^{-1}$ (Babadzhanov \& Kramer 1965).

In the comprehensive work undertaken by Dutch Meteor Society (DMS) and North Australian Planetary Observers - Meteor Section (NAPO-MS) in years 19811991 and described in detail by Jenniskens (1994) one can read about weak stream called $o$-Cygnids. During 98 hours of effective time of observations 8 observers noted 72 possible members of that stream. From this data Jenniskens (1994) estimated the following parameters of the stream:

- equatorial coordinates of the radiant during the maximum of activity: $\alpha=305^{\circ} \quad \delta=+47^{\circ}$,

- drift of the radiant (in units ${ }^{\circ} /$ day): $\Delta \alpha=+0.6 \quad \Delta \delta=$ +0.2 ,

- maximum of activity: $\lambda_{\odot(1950.0)}=116.0^{\circ} \pm 0.5^{\circ}$,

- population index $r=2.7$, where $r$ is defined as:

$r=\frac{\Phi(m+1)}{\Phi(m)}$

where

$\Phi(m)=\sum_{-\infty}^{m} N(m)$

and $N(m)$ is the number of meteors with magnitude $m$ corrected for probabilities of perception given by Koschack \& Rendtel (1990), 
Table 1. List of visual CMW observers

\begin{tabular}{|c|c|c|c|c|c|c|c|c|c|}
\hline \multirow[b]{2}{*}{ Observer } & \multicolumn{3}{|c|}{1995} & \multicolumn{3}{|c|}{$\overline{1996}$} & \multicolumn{3}{|c|}{$\overline{1997}$} \\
\hline & $t_{\text {eff }}$ & $N_{\alpha}$ & $N_{\mathrm{sp}}$ & $t_{\text {eff }}$ & $N_{\alpha}$ & $N_{\mathrm{sp}}$ & $t_{\text {eff }}$ & $N_{\alpha}$ & $N_{\mathrm{sp}}$ \\
\hline Maciej Reszelski & $\overline{56^{\mathrm{h}} 51^{\mathrm{m}}}$ & 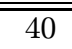 & $\overline{2778}$ & $27^{\mathrm{h}} 22^{\mathrm{m}}$ & 35 & $\overline{181}$ & $11^{\mathrm{h}} 58^{\mathrm{m}}$ & $\overline{18}$ & $\overline{114}$ \\
\hline Arkadiusz Olech & $32^{\mathrm{h}} 45^{\mathrm{m}}$ & 35 & 257 & $16^{\mathrm{h}} 55^{\mathrm{m}}$ & 19 & 129 & $31^{\mathrm{h}} 31^{\mathrm{m}}$ & 39 & 234 \\
\hline Tomasz Fajfer & - & - & - & $31^{\mathrm{h}} 30^{\mathrm{m}}$ & 33 & 134 & $39^{\mathrm{h}} 30^{\mathrm{m}}$ & 52 & 341 \\
\hline Konrad Szaruga & - & - & - & $11^{\mathrm{h}} 28^{\mathrm{m}}$ & 6 & 84 & $50^{\mathrm{h}} 16^{\mathrm{m}}$ & 85 & 257 \\
\hline Marcin Gajos & $26^{\mathrm{h}} 00^{\mathrm{m}}$ & 12 & 144 & $6^{\mathrm{h}} 18^{\mathrm{m}}$ & 4 & 38 & $10^{\mathrm{h}} 40^{\mathrm{m}}$ & 11 & 83 \\
\hline Krzysztof Socha & $17^{\mathrm{h}} 00^{\mathrm{m}}$ & 21 & 103 & $6^{\mathrm{h}} 54^{\mathrm{m}}$ & 4 & 38 & $18^{\mathrm{h}} 16^{\mathrm{m}}$ & 12 & 142 \\
\hline Maciej Kwinta & $4^{\mathrm{h}} 00^{\mathrm{m}}$ & 0 & 13 & $8^{\mathrm{h}} 10^{\mathrm{m}}$ & 0 & 13 & $26^{\mathrm{h}} 50^{\mathrm{m}}$ & 9 & 157 \\
\hline Krzysztof Wtorek & $5^{\mathrm{h}} 42^{\mathrm{m}}$ & 5 & 32 & $10^{\mathrm{h}} 00^{\mathrm{m}}$ & 14 & 67 & $12^{\mathrm{h}} 55^{\mathrm{m}}$ & 12 & 45 \\
\hline Łukasz Sanocki & $14^{\mathrm{h}} 30^{\mathrm{m}}$ & 17 & 62 & $5^{\mathrm{h}} 46^{\mathrm{m}}$ & 8 & 21 & $8^{\mathrm{h}} 04^{\mathrm{m}}$ & 5 & 27 \\
\hline Tomasz Dziubiński & $15^{\mathrm{h}} 47^{\mathrm{m}}$ & 16 & 35 & $3^{\mathrm{h}} 30^{\mathrm{m}}$ & 5 & 20 & $6^{\mathrm{h}} 00^{\mathrm{m}}$ & 3 & 25 \\
\hline Jarosław Dygos & - & - & - & - & - & - & $24^{\mathrm{h}} 30^{\mathrm{m}}$ & 16 & 157 \\
\hline Gracjan Maciejewski & - & - & - & - & - & - & $21^{\mathrm{h}} 15^{\mathrm{m}}$ & 8 & 91 \\
\hline Andrzej Skoczewski & - & - & - & - & - & - & $20^{\mathrm{h}} 23^{\mathrm{m}}$ & 28 & 85 \\
\hline Artur Szaruga & - & - & - & - & - & - & $19^{\mathrm{h}} 55^{\mathrm{m}}$ & 14 & 71 \\
\hline Wojciech Jonderko & - & - & - & $4^{\mathrm{h}} 15^{\mathrm{m}}$ & 0 & 11 & $15^{\mathrm{h}} 24^{\mathrm{m}}$ & 7 & 67 \\
\hline Marcin Konopka & - & - & - & - & - & - & $18^{\mathrm{h}} 39^{\mathrm{m}}$ & 17 & 181 \\
\hline Michał Jurek & - & - & - & $9^{\mathrm{h}} 31^{\mathrm{m}}$ & 9 & 80 & $7^{\mathrm{h}} 39^{\mathrm{m}}$ & 9 & 53 \\
\hline Tomasz Żywczak & - & - & - & - & - & - & $16^{\mathrm{h}} 38^{\mathrm{m}}$ & 10 & 43 \\
\hline Maria Woźniak & $13^{\mathrm{h}} 45^{\mathrm{m}}$ & 18 & 43 & - & - & - & & - & - \\
\hline Tomasz Ramza & - & - & - & $6^{\mathrm{h}} 30^{\mathrm{m}}$ & 5 & 49 & $5^{\mathrm{h}} 59^{\mathrm{m}}$ & 1 & 32 \\
\hline Łukasz Pospieszny & - & - & - & $9^{\mathrm{h}} 44^{\mathrm{m}}$ & 4 & 62 & $3^{\mathrm{h}} 31^{\mathrm{m}}$ & 1 & 18 \\
\hline Piotr Grzywacz & $11^{\mathrm{h}} 30^{\mathrm{m}}$ & 21 & 55 & - & - & - & - & - & - \\
\hline Robert Szczerba & - & - & - & $5^{\mathrm{h}} 00^{\mathrm{m}}$ & 6 & 21 & $6^{\mathrm{h}} 20^{\mathrm{m}}$ & 1 & 100 \\
\hline Krzysztof Kamiński & - & - & - & - & - & - & $8^{\mathrm{h}} 47^{\mathrm{m}}$ & 6 & 60 \\
\hline Łukasz Raurowicz & - & - & - & $1^{\mathrm{h}} 07^{\mathrm{m}}$ & 2 & 3 & $6^{\mathrm{h}} 16^{\mathrm{m}}$ & 6 & 44 \\
\hline Tadeusz Sobczak & - & - & - & - & - & - & $6^{\mathrm{h}} 10^{\mathrm{m}}$ & 20 & 46 \\
\hline Rafał Kopacki & - & - & - & $5^{\mathrm{h}} 30^{\mathrm{m}}$ & 8 & 45 & - & - & - \\
\hline Michał Marek & - & - & - & $4^{\mathrm{h}} 00^{\mathrm{m}}$ & 6 & 5 & $0^{\mathrm{h}} 30^{\mathrm{m}}$ & 1 & 4 \\
\hline Elżbieta Brembor & $4^{\mathrm{h}} 00^{\mathrm{m}}$ & 8 & 5 & & - & - & - & - & - \\
\hline Ireneusz Sławiński & - & - & - & $3^{\mathrm{h}} 00^{\mathrm{m}}$ & 0 & 12 & - & - & - \\
\hline Krzysztof Gdula & - & - & - & $2^{\mathrm{h}} 30^{\mathrm{m}}$ & 2 & 11 & - & - & - \\
\hline Marek Piotrowski & - & - & - & - & - & - & $2^{\mathrm{h}} 25^{\mathrm{m}}$ & 2 & 14 \\
\hline Paweł Trybus & - & - & - & - & - & - & $2^{\mathrm{h}} 00^{\mathrm{m}}$ & 0 & 10 \\
\hline Paweł Musialski & - & - & - & $1^{\mathrm{h}} 30$ & 1 & 7 & - & - & - \\
\hline Paweł Gembara & $1^{\mathrm{h}} 00^{\mathrm{m}}$ & 0 & 10 & - & - & - & - & - & - \\
\hline Total & $202^{\mathrm{h}} 50^{\mathrm{m}}$ & 193 & 1037 & $180^{\mathrm{h}} 30^{\mathrm{m}}$ & 171 & 1031 & $402^{\mathrm{h}} 21^{\mathrm{m}}$ & 393 & 2501 \\
\hline
\end{tabular}

- Maximal Zenithal Hourly Rates $(Z H R \mathrm{~s})$ are equal to $2.5 \pm 0.8$, where $Z H R$ is defined as:

$Z H R=\frac{N_{\mathrm{h}} \cdot r^{(6.5-L M)}}{(\sin H)^{\gamma}}$

where $N_{\mathrm{h}}$ is the observed number of meteors per hour (corrected for clouds coverage), $L M$ is the limiting magnitude in the field of view, $H$ is an altitude of the radiant of the stream, and $\gamma$ is a zenith exponent factor,

- geocentric velocity: $V_{\infty}=37 \mathrm{~km} \mathrm{~s}^{-1}$.

From the Fig. 11 in paper by Jenniskens (1994) it is clearly visible that the activity of $o$-Cygnids lasts from $\lambda_{\odot} \approx 105^{\circ}$ to $\lambda_{\odot} \approx 127^{\circ}$ i.e. from around July 5 th to July 27 th.

\section{Observations}

\subsection{Visual observations}

July is a good month for meteor observers in Poland. Polish observers associated in Comets and Meteors Workshop (CMW) are mostly young people studying at secondary schools or universities. July is the first month of summer holidays. The warm nights, a lot of free time, good weather conditions strongly encourage to observations. Finally about $60-80 \%$ of whole year observations made by CMW members are collected in July and August.

All above facts and rapidly growing interest in meteor observations in Poland during the last few years allowed us to investigate the activity of the stream called $\alpha$-Cygnids or o-Cygnids. Each July in years 1995-1997 many meteor 
Table 2. List of telescopic CMW observers

\begin{tabular}{||l||r|r|r||r|r|r||r|r|r||}
\hline \multirow{2}{*}{ Observer } & \multicolumn{3}{c||}{1996} & \multicolumn{3}{c||}{1997} & \multicolumn{3}{c||}{ Total } \\
\cline { 2 - 9 } & $t_{\text {eff }}$ & $N_{\alpha}$ & $N_{\mathrm{sp}}$ & $t_{\mathrm{eff}}$ & $N_{\alpha}$ & $N_{\mathrm{sp}}$ & $t_{\mathrm{eff}}$ & $N_{\alpha}$ & $N_{\mathrm{sp}}$ \\
\hline \hline Tomasz Dziubiński & $4^{\mathrm{h}} 51^{\mathrm{m}}$ & 7 & 20 & $4^{\mathrm{h}} 40^{\mathrm{m}}$ & 5 & 31 & $9^{\mathrm{h}} 31^{\mathrm{m}}$ & 12 & 51 \\
Michał Jurek & $1^{\mathrm{h}} 56^{\mathrm{m}}$ & 1 & 3 & $3^{\mathrm{h}} 31^{\mathrm{m}}$ & 1 & 13 & $5^{\mathrm{h}} 27^{\mathrm{m}}$ & 2 & 16 \\
Konrad Szaruga & $1^{\mathrm{h}} 21^{\mathrm{m}}$ & 3 & 5 & $3^{\mathrm{h}} 58^{\mathrm{m}}$ & 6 & 34 & $5^{\mathrm{h}} 19^{\mathrm{m}}$ & 9 & 39 \\
Marcin Gajos & $1^{\mathrm{h}} 49^{\mathrm{m}}$ & 1 & 10 & $2^{\mathrm{h}} 42^{\mathrm{m}}$ & 4 & 17 & $4^{\mathrm{h}} 31^{\mathrm{m}}$ & 5 & 27 \\
Jarosław Dygos & - & - & - & $4^{\mathrm{h}} 09^{\mathrm{m}}$ & 1 & 10 & $4^{\mathrm{h}} 09^{\mathrm{m}}$ & 1 & 10 \\
Tomasz Fajfer & $2^{0} 5^{\mathrm{m}}$ & 2 & 9 & $2^{\mathrm{h}} 00^{\mathrm{m}}$ & 2 & 9 & $4^{\mathrm{h}} 05^{\mathrm{m}}$ & 4 & 18 \\
Marcin Konopka & - & - & - & $3^{\mathrm{h}} 31^{\mathrm{m}}$ & 1 & 13 & $3^{\mathrm{h}} 31^{\mathrm{m}}$ & 1 & 13 \\
Wojciech Jonderko & - & - & - & $1^{\mathrm{h}} 23^{\mathrm{m}}$ & 0 & 0 & $1^{\mathrm{h}} 23^{\mathrm{m}}$ & 0 & 0 \\
Eukasz Pospieszny & - & - & - & $1^{\mathrm{h}} 11^{\mathrm{m}}$ & 1 & 3 & $1^{\mathrm{h}} 11^{\mathrm{m}}$ & 1 & 3 \\
Rafał Kopacki & $1^{\mathrm{h}} 00^{\mathrm{m}}$ & 0 & 3 & - & - & - & $1^{\mathrm{h}} 00^{\mathrm{m}}$ & 0 & 3 \\
Krzysztof Wtorek & $1^{\mathrm{h}} 00^{\mathrm{m}}$ & 0 & 3 & - & - & - & $1^{\mathrm{h}} 00^{\mathrm{m}}$ & 0 & 3 \\
Maciej Reszelski & - & - & - & $0^{\mathrm{h}} 59^{\mathrm{m}}$ & 4 & 6 & $0^{\mathrm{h}} 59^{\mathrm{m}}$ & 4 & 6 \\
Michał Kopczak & $0^{\mathrm{h}} 53^{\mathrm{m}}$ & 1 & 1 & - & - & - & $0^{\mathrm{h}} 53^{\mathrm{m}}$ & 1 & 1 \\
Andrzej Skoczewski & - & - & - & $0^{\mathrm{h}} 44^{\mathrm{m}}$ & 0 & 4 & $0^{\mathrm{h}} 44^{\mathrm{m}}$ & 0 & 4 \\
\hline Total & $14^{\mathrm{h}} 55^{\mathrm{m}}$ & 15 & 54 & $28^{\mathrm{h}} 32^{\mathrm{m}}$ & 26 & 139 & $43^{\mathrm{h}} 27^{\mathrm{m}}$ & 41 & 193 \\
\hline \hline
\end{tabular}

observers watched the sky using visual, telescopic and photographic techniques. In order to obtain most reliable results we had to remove observations made incorrectly or in poor conditions. Using our standard methods (Olech \& Woźniak 1996; Olech 1997) we required that:

- mean limiting magnitude (LM) in the field of view should be at least $5.0 \mathrm{mag}$,

- cloud coverage correction factor $F$ should be smaller than 1.7 ,

- the radiant of the stream should be above $20^{\circ}$ over the horizon (in Polish latitudes during whole July this condition is always satisfied),

- the center of the field of view should be at an altitude higher than $40^{\circ}$.

Finally we obtained $785^{\mathrm{h}} 41^{\mathrm{m}}$ of effective time of visual observations. During this time a group of $35 \mathrm{CMW}$ members noted 757 possible meteors from $\alpha$-Cygnid stream and 4569 sporadic meteors. More detailed statistics with names of CMW observers is given in Table 1.

\subsection{Telescopic observations}

Telescopic observations present a very useful tool for meteor investigators. Meteors are very often plotted with a larger accuracy than in case of visual observations. It gives the possibility to study the structure and drift of the radiant. We also obtain information about magnitude distribution for fainter events. The main problem with telescopic observations is that this kind of watching meteors requires good equipment (preferably binoculars with a large field of view mounted on tripod), experienced observers and a lot of patience.

Fortunately July is usually the time in which we organize an Astronomical Camp of CMW, which takes place at the Observational Station of Warsaw University Observatory in Ostrowik. The number of participants is always around 15, so we organize two four persons groups observing visually, one or two persons working with a few cameras pointed at different directions, and threefour persons observing telescopically different fields located $10^{\circ}-40^{\circ}$ from the supposed radiant of $\alpha$-Cygnids. We used mostly $7 \times 50,10 \times 50$ and $20 \times 60$ binoculars and Russian AT- 1 refractors $(5 \times 50$, field of view as large as $11^{\circ}$ ). For plotting meteors we use $A$-type maps of International Meteor Organization (IMO) or Uranometria 2000.0 charts. Of course other observers which do not participate in the camp observe $\alpha$-Cygnids both visually and telescopically at their locations.

Finally 14, our observers obtained $43^{\mathrm{h}} 27^{\mathrm{m}}$ of telescopic observations with 234 meteors detected. The number of possible $\alpha$-Cygnids is equal to 41 . Table 2 summarizes our telescopic observations.

\section{Results}

\subsection{Radiant of $\alpha$-Cygnids}

During July of 1995, 1996 and 1997 CMW observers plotted on gnomonic star maps 2748 paths of meteor events. For each of them the angular velocity was estimated. We used $0-5$ scale with 0 corresponding to stationary meteor, 1 to very slow event, 2 to slow, 3 to medium, 4 to fast and 5 to very fast meteor. Equatorial coordinates of the begins and ends of these events and their velocities were put into 
Table 3. Magnitude distribution for 1995-1997 $\alpha$-Cygnids

\begin{tabular}{|l|ccccccccccccc|c|}
\hline \hline Year & $\leq-5$ & -4 & -3 & -2 & -1 & 0 & 1 & 2 & 3 & 4 & 5 & 6 & 7 & Tot. \\
\hline \hline 1995 & 0 & 0 & 3.5 & 1.5 & 4 & 5 & 32.5 & 72.5 & 35 & 23 & 14 & 2 & 0 & 193 \\
1996 & 0 & 0 & 0.5 & 3.5 & 4 & 7.5 & 13 & 25 & 45.5 & 42 & 25 & 5 & 0 & 171 \\
1997 & 0 & 3 & 0.5 & 2.5 & 6 & 18.5 & 32 & 65.5 & 93 & 81.5 & 53 & 18.5 & 0 & 374 \\
\hline Tot. & 0 & 3 & 4.5 & 7.5 & 14 & 31 & 77.5 & 163 & 173.5 & 146.5 & 92 & 25.5 & 0 & 738 \\
\hline \hline
\end{tabular}

Table 4. Magnitude distribution for 1995-1997 sporadics

\begin{tabular}{|c|c|c|c|c|c|c|c|c|c|c|c|c|c|c|}
\hline Year & $\bar{c} \leq-5$ & $\overline{-14}$ & -3 & -2 & -1 & $\overline{0}$ & 1 & 2 & $\overline{3}$ & $\overline{4}$ & $\overline{5}$ & $\overline{6}$ & $\overline{7}$ & Tot. \\
\hline$\overline{1995}$ & $\overline{\overline{0}}$ & $\overline{1}$ & 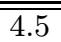 & 6 & $\overline{16}$ & $\bar{~} 62.5$ & $\bar{~} 139.5$ & 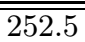 & 268.5 & $\overline{\overline{173}}$ & $\overline{87.5}$ & 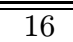 & $\overline{\overline{0}}$ & $\bar{~} 1027$ \\
\hline 1996 & 0 & 0 & 4.5 & 10 & 32 & 72.5 & 123.5 & 197.5 & 239 & 202.5 & 132 & 17.5 & 0 & 1031 \\
\hline 1997 & 5 & 16 & 11 & 25 & 67.5 & 123 & 274.5 & 405.5 & 547.5 & 577.5 & 348.5 & 86.5 & 0.5 & 2488 \\
\hline Tot. & 5 & 17 & 20 & 41 & 115.5 & 258 & 537.5 & 855.5 & 1055 & 953 & 568 & 120 & 0.5 & 4546 \\
\hline
\end{tabular}

Table 5. Magnitude distribution for 1996-1997 telescopic $\alpha$-Cygnids

\begin{tabular}{|c|cccccc|c|}
\hline \hline Year & 4 & 5 & 6 & 7 & 8 & 9 & Tot. \\
\hline \hline 1996 & 0.5 & 0.5 & 6 & 6.5 & 1.5 & 0 & 15 \\
1997 & 0 & 2 & 3 & 8.5 & 7.5 & 5 & 26 \\
\hline Tot. & 0.5 & 2.5 & 9 & 15 & 9 & 5 & 41 \\
\hline \hline
\end{tabular}

the RADIANT software (Arlt 1992). This software as an input also requires the geocentric velocity of the meteors $V_{\infty}$ and the daily drift of the radiant. Changing both these values we can obtain different density distributions of the probability area near suspected radiant. Choosing the best distribution (this one with smallest $\chi^{2}$ parameter) we are able to estimate the values of $V_{\infty}$ and the daily drift. The systematic errors play a role, which are difficult to handle and estimate of the accuracy of the obtained value of $V_{\infty}$ is difficult but the errors are at minimum $\pm 5 \mathrm{~km} \mathrm{~s}^{-1}$. For more details see Arlt (1993).

Before analyzing our sample we decided to analyze also the meteors observed by Denning (1919). However we selected only meteors observed by him during July nights. Number of these events accounted to 20. We performed our calculation using parameters of the stream given by Jenniskens (1994) i.e. $V_{\infty}=37 \mathrm{~km} \mathrm{~s}^{-1}, \lambda_{\odot(\max )}=116^{\circ}$, $\Delta \alpha=+0.6$ and $\Delta \delta=+0.2$. Results as probability function distribution of the presence of radiant are presented in Fig. 1. The best fit of the two dimensional Gaussian surface to the density of probability map gives coordinates of the radiant equal to $\alpha=312.4^{\circ}$ and $\delta=+48.4^{\circ}$. The accuracy of this estimate is certainly low due to the small number of events observed by Denning (1919).

Fortunately, the sample collected by CMW observers in years 1995-1997 is significantly larger. It allows us to derive a few valuable conclusions. First we calculate our sample (2748 meteors including possible members of the stream, sporadics and meteors from other showers) using parameters given by Jenniskens (1994). During calculation we remove meteors observed at a distance larger than $85^{\circ}$

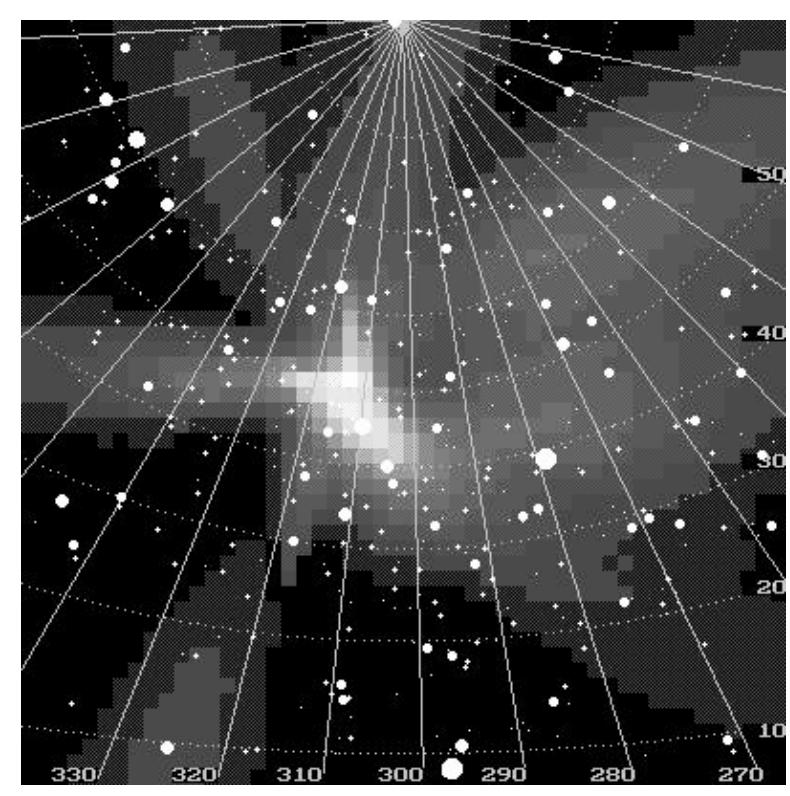

Fig. 1. The radiant of $\alpha$-Cygnids resulting from Denning's (1919) observations. Assumed parameters are: $V_{\infty}=$ $37 \mathrm{~km} \mathrm{~s}^{-1}, \lambda_{\odot(\max )}=116^{\circ}, \Delta \alpha=+0.6^{\circ}$ and $\Delta \delta=+0.2^{\circ}$. Number of the events is 20

from the radiant of the stream. The prominence of the $\alpha$-Cygnid radiant on the resulting picture is striking. The best fit gives coordinates of the radiant as $\alpha=302.0^{\circ}$ and $\delta=+46.1^{\circ}$. Nevertheless we obtain better results i.e. a more compact shape of the radiant using geocentric velocity $V_{\infty}=41 \mathrm{~km} \mathrm{~s}^{-1}$ and the drift of the radiant $\Delta \alpha=+0.6, \Delta \delta=+0.2$. The resulting radiant picture for 
Table 6. Magnitude distribution for 1996-1997 telescopic sporadics

\begin{tabular}{|l|cccccccccc|c|}
\hline \hline Year & 1 & 2 & 3 & 4 & 5 & 6 & 7 & 8 & 9 & 10 & Tot. \\
\hline \hline 1996 & 0 & 0.5 & 3 & 1 & 6.5 & 8.5 & 24.5 & 9 & 1 & 0 & 54 \\
1997 & 1 & 0.5 & 2.5 & 6 & 5.5 & 18.5 & 51.5 & 39.5 & 12 & 2 & 139 \\
\hline Tot. & 1 & 1 & 5.5 & 7 & 12 & 27 & 76 & 48.5 & 13 & 2 & 193 \\
\hline \hline
\end{tabular}

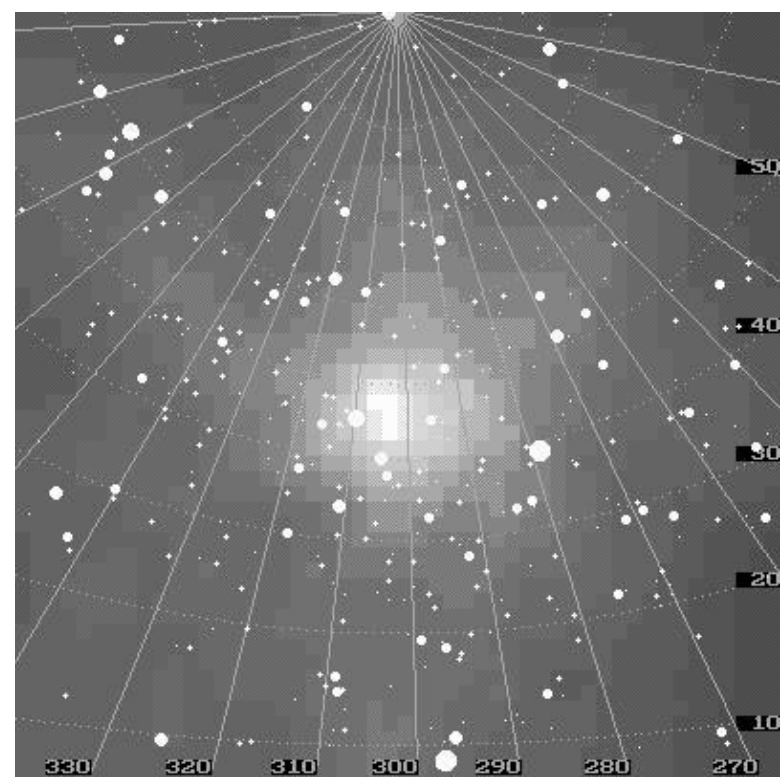

Fig. 2. The radiant of $\alpha$-Cygnids resulting from CMW visual data. Assumed parameters are: $V_{\infty}=41 \mathrm{~km} \mathrm{~s}^{-1}, \lambda_{\odot(\max )}=$ $116^{\circ}, \Delta \alpha=+0.6^{\circ}$ and $\Delta \delta=+0.2^{\circ}$. Number of the events is 2748

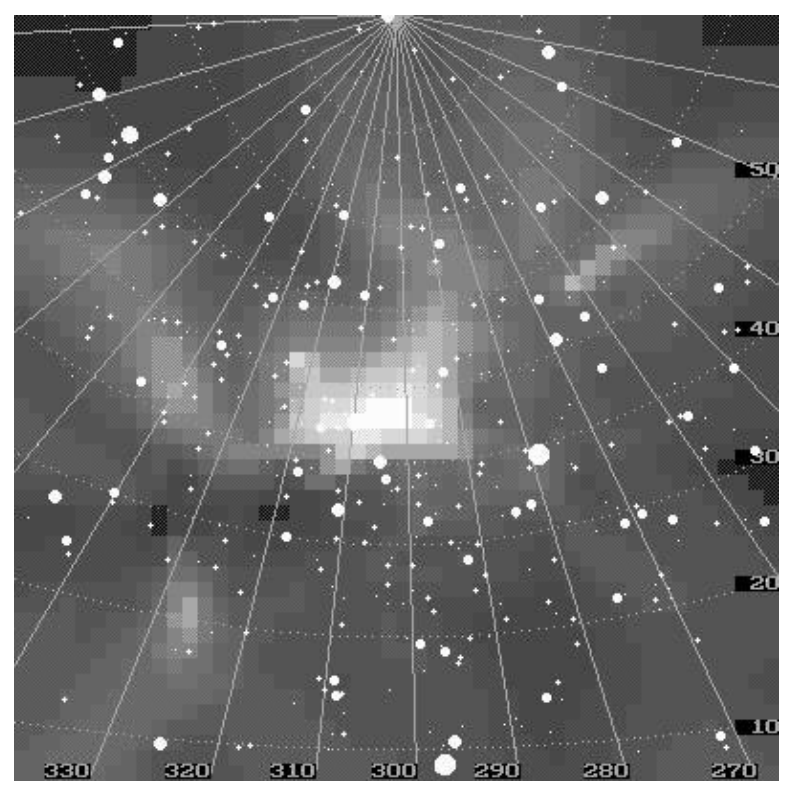

Fig. 3. The radiant of $\alpha$-Cygnids resulting from CMW telescopic data. Used parameters are: $V_{\infty}=40 \mathrm{~km} \mathrm{~s}^{-1}, \lambda_{\odot(\max )}=$ $116^{\circ}, \Delta \alpha=+0.6^{\circ}$ and $\Delta \delta=+0.2^{\circ}$. Number of the events is 234 the above parameters is displayed in Fig. 2. The final coordinates of the radiant of $\alpha$-Cygnid stream are $\alpha=302.5^{\circ}$ and $\delta=+46.3^{\circ}$, which do not differ significantly from coordinates obtained for parameters given by Jenniskens (1994).

We also used the RADIANT software for the analysis of the paths of our telescopic meteors. Our sample contains 234 meteors with known paths and velocities. The resulting density distribution from telescopic observations is displayed in Fig. 3. The best fit (with smallest $\chi^{2}$ value) is obtained for the following parameters: geocentric velocity $V_{\infty}=40 \mathrm{~km} \mathrm{~s}^{-1}$, the daily drift of the radiant $\Delta \alpha=+0.6^{\circ}$ and $\Delta \delta=+0.2^{\circ}$. The coordinates of the center of the radiant are $\alpha=304.9^{\circ}$ and $\delta=+46.2^{\circ}$. One can see that the position of the radiant obtained from telescopic observations differs from the position obtained from visual data by only $1.7^{\circ}$. Taking into account that radii of the majority of radiants vary between $2^{\circ}$ and $7^{\circ}$ both our results are strictly consistent. It is also clear that our parameters are in very good agreement with the data of the one photographed meteor (Babadzhanov \& Kramer 1965).

\subsection{Population index $r$}

In years 1995-1997 the CMW observers made as many as 738 and 4546 estimates of the brightness of meteor events from $\alpha$-Cygnids and sporadics, respectively. The distribution of this quantity is presented in Tables 3 and 4 .

Such a large amount of magnitude estimates for $\alpha$ Cygnids encouraged us to compute the value of the population index $r$ defined in Eq. (1). We obtained $r=$ $2.55 \pm 0.14$ which is a typical value among meteor streams. Jenniskens (1994) obtained a similar result with $r$ equal to 2.7. The population index obtained from the magnitudes of our 4546 sporadics is equal to $r=2.61 \pm 0.23$.

Also the telescopic observers estimated the magnitudes of meteor events. The magnitude distributions for 1996 and $1997 \alpha$-Cygnids and sporadics are presented in Tables 5 and 6.

\subsection{Activity profile}

Knowing the value of $r$ we can compute $Z H R$ using the formula given in (3). According to the results of Koschack (1994) and Bellot (1995) who showed that for visual observations with radiant altitudes higher than $20^{\circ}$ the zenith exponent factor $\gamma \approx 1.0$, we adopted $\gamma=1.0$. 


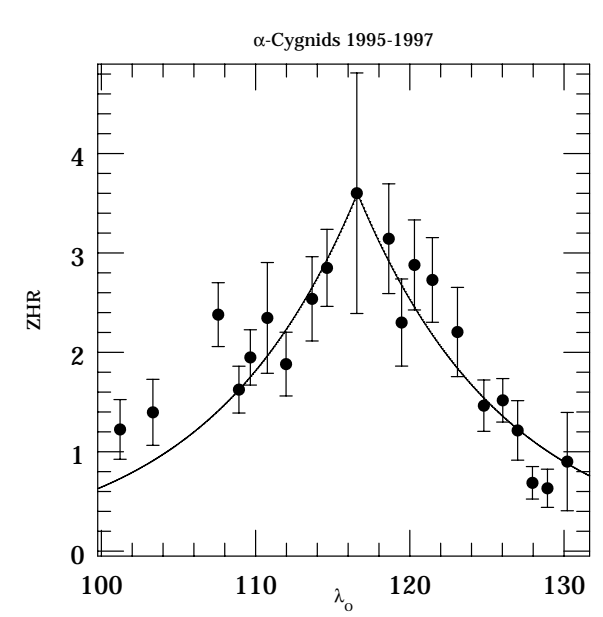

Fig. 4. The activity profile of $\alpha$-Cygnids during 1995-1997. The solid line represents the fit given in Eq. (4)

The resulting activity profile of $\alpha$-Cygnids in years 1995-1997 is exhibited in Fig. 4. The activity of the stream lasts from $\lambda_{\odot} \approx 100^{\circ}$ (June 30) to $\lambda_{\odot} \approx 130^{\circ}$ (July 31). It seems to be slightly wider than the result of Jenniskens (1994) who noted meteors from $\alpha$-Cygnid stream in interval $\lambda_{\odot}=105-127^{\circ}$. The accuracy of the $Z H R$ estimates by Jenniskens (1994) was low due to the small number of his observations, therefore we prefer our result.

Our Fig. 4 one exhibits a clear maximum of activity at $\lambda_{\odot} \approx 116.5^{\circ}$ with $Z H R=3.6 \pm 1.2$. The error of this estimate is large but points in the vicinity of the maximum have smaller errors and their moments and $Z H R \mathrm{~s}$ are $\lambda_{\odot}=114.5^{\circ}$ with $Z H R=2.9 \pm 0.4$ and $\lambda_{\odot}=118.5^{\circ}$ with $Z H R=3.1 \pm 0.6$

The moment of the maximum and its $Z H R$ is in very good agreement with result of Jenniskens (1994) who obtained $\lambda_{\odot(\max )}=116.0 \pm 0.5^{\circ}$ with $Z H R_{\max }=2.5 \pm 0.8$.

Jenniskens (1994) found also that the activity profiles of meteor streams are well represented by the following equation:

$Z H R=Z H R_{\max } \times 10^{-B \cdot\left|\lambda_{\odot}-\lambda_{\odot \max }\right|}$.

For $\alpha$-Cygnids he found $B=0.13 \pm 0.03$. As we have already written our activity profile is much broader and thus our value of $B$ is smaller and equal to $0.045 \pm 0.005$. In Fig. 4 the solid line represents fit given in Eq. (4) with $Z H R_{\max }=3.6, \lambda_{\odot \max }=116.5^{\circ}$ and $B=0.045$.

\subsection{Velocity distribution}

The CMW observers estimated also the angular velocity of the events. The $0-5$ scale (defined in Sect. 3.1 of this paper) was used. Finally we obtained 754 estimates of the angular velocity for $\alpha$-Cygnids and 4339 estimates for sporadics. The velocity distribution from visual observations is presented in Tables 7-8.
Table 7. Velocity distribution for 1995-1997 $\alpha$-Cygnids

\begin{tabular}{|l|cccccc|c|}
\hline \hline Year & 0 & 1 & 2 & 3 & 4 & 5 & Tot. \\
\hline \hline 1995 & 0 & 0 & 6 & 63 & 96 & 28 & 193 \\
1996 & 0 & 0 & 9 & 44 & 97 & 20 & 170 \\
1997 & 4 & 2 & 22 & 166 & 172 & 25 & 391 \\
\hline Tot. & 4 & 2 & 37 & 273 & 365 & 73 & 754 \\
\hline \hline
\end{tabular}

Table 8. Velocity distribution for 1995-1997 sporadics

\begin{tabular}{|l|cccccc|r|}
\hline \hline Year & 0 & 1 & 2 & 3 & 4 & 5 & Tot. \\
\hline \hline 1995 & 6 & 17 & 68 & 247 & 418 & 171 & 927 \\
1996 & 4 & 22 & 86 & 243 & 433 & 233 & 1021 \\
1997 & 42 & 31 & 202 & 710 & 938 & 468 & 2391 \\
\hline Tot. & 52 & 70 & 356 & 1200 & 1789 & 872 & 4339 \\
\hline \hline
\end{tabular}

Table 9. Velocity distribution for 1996-1997 telescopic $\alpha$-Cygnids

\begin{tabular}{|l|ccccccc|c|}
\hline \hline Year & 0 & A & B & C & D & E & F & Tot. \\
\hline \hline 1996 & 0 & 0 & 1 & 6 & 6 & 2 & 0 & 15 \\
1997 & 0 & 0 & 4 & 5 & 11 & 4 & 2 & 26 \\
\hline Tot. & 0 & 0 & 5 & 11 & 17 & 6 & 2 & 41 \\
\hline \hline
\end{tabular}

We used the above distributions to find another proof for existence of the $\alpha$-Cygnid stream. We compared empirical velocity distributions of $\alpha$-Cygnids and sporadics using Kolmogorov-Smirnov and $\chi^{2}$ tests. We obtained that with the probability larger than 0.999 both distributions are different. Such a large probability is certainly caused by the clear enhancement of meteors with velocity 3 and 4 in $\alpha$-Cygnid velocity distribution. This result is also is good agreement with the value of geocentric velocity obtained from RADIANT analysis of our visual and telescopic data. The meteors with velocity $V_{\infty}=40-41 \mathrm{~km} \mathrm{~s}^{-1}$ given by RADIANT software at mean distance from the radiant of the stream appear mainly with velocities 3 and 4 in $0-5$ scale.

The velocity of meteor events was also estimated by our telescopic observers. They used $A-F$ scale with $A$ corresponding to the angular velocity $2^{\circ} / \mathrm{s}$ and $F$ to over $25^{\circ} / \mathrm{s}$. Finally we obtained 41 estimates of the angular velocity for telescopic $\alpha$-Cygnids and 192 velocity estimates for telescopic sporadics. Both distributions are presented in Tables 9-10.

For telescopic observations the distance from the radiant is generally well defined. Usually it is worthwhile to analyze the mean angular velocity as a function of distance from the radiant. Unfortunately due to the small number of our telescopic $\alpha$-Cygnids which were observed in as many as 10 fields such an analysis is impossible yet. 
Table 10. Velocity distribution for 1996-1997 telescopic sporadics

\begin{tabular}{|l|ccccccc|r|}
\hline \hline Year & 0 & A & B & C & D & E & F & Tot. \\
\hline \hline 1996 & 1 & 1 & 6 & 13 & 19 & 10 & 4 & 54 \\
1997 & 2 & 4 & 11 & 31 & 43 & 30 & 17 & 138 \\
\hline Tot. & 3 & 5 & 17 & 44 & 62 & 40 & 21 & 192 \\
\hline \hline
\end{tabular}

\section{Discussion}

We presented here the results of visual and telescopic observations of $\alpha$-Cygnid stream made by CMW observers in years 1995-1997. This stream is not included in the IMO List of Visual Meteor Showers (Rendtel et al. 1995) due to the lack of photographic, video and radio data confirming its presence. The only continuous (not visual) surveys are the Harvard Super Schmidt Program of the 1952-54 period and the Harvard Radar Project in the 1960's. Both have gaps in coverage due to weather or instrument irregularities. Unfortunately the $\alpha$-Cygnids seem to fall in such gaps. McCrosky \& Posen (1961) presented the orbital elements of 2529 meteors photographed simultaneously from two camera stations of the Harvard Meteor Project. The mean number of meteors captured in periods January-June and August-December is 221 events per month. The number of meteors photographed in July is only 102. It is over two times smaller than in other months and certainly it is the reason of lacking the meteors from $\alpha$-Cygnid stream in that project. The similar situation occurred during the Harvard Radar Project. However recent radio results obtained by Michael Boschat from Dalhousie University in Canada (Boschat 1998) showed clear enhancement of radio echoes in days 1998 July 19-20.

Analysis of 2748 paths of meteor events observed in July 1995, 1996 and 1997 allowed us to obtain the basic properties of the stream. The geocentric velocity of the $\alpha$-Cygnid events is $V_{\infty}=41 \mathrm{~km} \mathrm{~s}^{-1}$ and daily drift of the radiant $\Delta \alpha=+0.6^{\circ}, \Delta \delta=+0.2^{\circ}$. The coordinates of the center of the radiant of $\alpha$-Cygnid stream are $\alpha=302.5^{\circ}$ and $\delta=+46.3^{\circ}$.

We performed a similar analysis for 234 telescopic meteors plotted also by CMW observers. The parameters of the stream are in very good agreement with the results derived from visual data.

Our results are also consistent with visual observations by Denning (1919) and Jenniskens (1994) and the photographic results obtained by Babadzhanov \& Kramer (1965).
From magnitude distribution of $738 \alpha$-Cygnids we obtained the population index $r$ equal to $2.55 \pm 0.14$ which is in good agreement with previously obtained value $r=2.7$ (Jenniskens 1994).

The velocity distributions of $754 \alpha$-Cygnids and 4339 sporadics are different, with a probability higher than 0.999 which gives another proof for reality of the $\alpha$-Cygnid stream.

From visual observations made by CMW members during the years 1995-1997 we obtained the clear activity profile of the $\alpha$-Cygnid stream. Meteors belonging to this shower were detected from June 30 to July 31 with clear maximum near July $18\left(\lambda_{\odot}=116.5^{\circ}\right)$. The maximal $Z H R$ s reached the level $3.6 \pm 1.2$. These results are in very good agreement with results presented by Jenniskens (1994) who obtained $\lambda_{\odot(\max )}=116.0 \pm 0.5^{\circ}$ and $Z H R_{\max }=2.5 \pm 0.8$.

In spite of making many photographic exposures we still have no photographic confirmation of this stream. To confirm of disprove our results further visual, telescopic and particularly video and photographic observations are clearly needed.

Acknowledgements. We would like to thank to all observers who sent us their observations. We are especially grateful to Prof. Jerzy Madej for helpful discussions, reading and commenting on the manuscript and also to Dr. Jacek Chołoniewski for many helpful hints. This work was supported by KBN grants 2 P03D 02011 and 2 P03D 00215 to A. Olech.

\section{References}

Arlt R., 1992, WGN 20, 62

Arlt R., 1993, Radiant 1.4 - Manual, p. 11

Babadzhanov P.B., Kramer E.N., 1965, Smits. Contr. to Astrophys. 11, 67

Bellot R.L.R., 1995, A\&A 301, 602

Boschat M., 1998 (private communication)

Denning W.F., 1919, J. British Astron. Assoc. 29, 161

Jenniskens P., 1994, A\&A 287, 990

Koschack R., Rendtel J., 1990, WGN 18, 54

Koschack R., 1994, Proceedings IMC Belogradchick, Knoefel A., Roggemans P. (eds.), p. 31

Kosinski J.W., 1990, V Zestawienie Rojów Meteorów, in Polish

McCrosky R.E., Posen A., 1961, Smiths. Contr. Astrophys. 4, 15

Olech A., Woźniak P., 1996, Earth Moon and Planets 73, 157

Olech A., 1997, A\&A 325, 1249

Rendtel J., Arlt R., Koschack R., et al., 1995, in "Handbook for Visual Meteor Observers", Rendtel J., Arlt R., and McBeath A., (eds.). International Meteor Organization, p. 126 